\title{
Trombose, Hemorragia e Trombocitopenia Induzidas pelas Vacinas contra a COVID-19: Protocolo de Atuação
}

\author{
Guidelines on COVID-19 Vaccine Induced Thrombosis, \\ Bleeding, and Thrombocytopenia
}

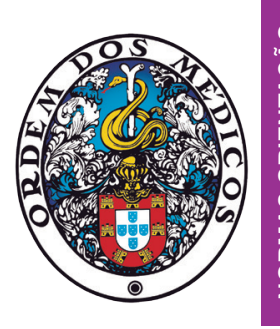

\author{
Sara MORAIS $\triangle 1,2$, Eugénia CRUZ 1,3 \\ Acta Med Port 2021 Sep;34(9):625-629 - https://doi.org/10.20344/amp.16602
}

\begin{abstract}
RESUMO
Após a generalização da vacinação contra a COVID-19, foram relatados efeitos adversos como trombose, hemorragia e trombocitopenia. Recentemente, após vacinação, foi reconhecido um síndrome raro e com mortalidade elevada, caraterizado por uma combinação não usual de trombocitopenia e trombose, em particular trombose dos seios venosos cerebrais, com muitas semelhanças com a trombocitopenia induzida pela heparina. Foram desenvolvidas diferentes recomendações na definição, diagnóstico e tratamento destas raras complicações. Apresentamos aqui, um protocolo de atuação baseado na evidência atual.
\end{abstract}

Palavras-chave: Vacinas Anti-COVID-19; Hemorragia; Trombocitopenia; Trombose

\section{ABSTRACT}

After widespread vaccination with COVID-19 vaccines, there have been worldwide reports on thrombosis, bleeding, and thrombocytopenia. Recently, a rare syndrome with a high mortality rate consisting of an unusual combination of thrombocytopenia and thrombosis, in particular cerebral venous sinus thrombosis, which clinically resembles heparin-induced thrombocytopenia, was reported following vaccination. Different statements and recommendations were developed regarding the definition, diagnosis, and treatment of these rare complications. We present here a protocol with recommendations, based on current evidence.

Keywords: COVID-19 Vaccines; Hemorrhage; Thrombocytopenia; Thrombosis

\section{INTRODUÇÃO}

A infeção pelo SARS-CoV-2 (COVID-19), que se associa a considerável morbilidade e mortalidade, tornou-se rapidamente numa pandemia global com impacto na saúde das populações e na economia de cada país. Tal como para outras infeções, a vacinação parece ser a principal forma de controlo da doença, pelo que desde cedo foi grande o esforço no sentido do desenvolvimento de vacinas, sendo que algumas destas já se encontram licenciadas e em uso generalizado.

Foi precisamente a generalização da vacinação que fez emergir novos efeitos adversos, e o aparecimento dos primeiros relatos de associação das vacinas baseadas no RNA mensageiro (RNAm) com trombocitopenia grave e hemorragia, mas sem trombose. ${ }^{1}$ Logo no início de janeiro, foi relatado nos EUA o caso de um profissional de saúde que, 16 dias após inoculação com a vacina produzida pela Pfizer-BioNTech, apresentou uma hemorragia fatal associada a trombocitopenia imune. ${ }^{1}$ Seguiu-se imediatamente a identificação de vários casos de trombocitopenia, com resposta favorável a terapias dirigidas à trombocitopenia imune [corticóides e imunoglobulina humana IV (IgIV)]. ${ }^{2}$ Atualmente, os casos de 'trombocitopenia' ou 'trombocitopenia imune'(PTI), com ou sem associação a hemorragia e mortalidade, são transversais às quatro vacinas aprovadas pela European Medicines Agency - EMA, e têm sido reportados quer na Vaccine Adverse Event Reporting System (VAERS) nos Estados Unidos, quer na EudraVigilance na
Europa, quer no MHRA Yellow Card no Reino Unido, entre outros. Aliás a PTI é uma complicação já bem conhecida, embora rara, de várias outras vacinas. ${ }^{3}$

No início de março de 2021, na Europa, começaram a surgir preocupações sobre o aparecimento de eventos trombóticos não usuais na sequência da vacinação com a vacina ChAdOx1 nCoV-19 da AstraZeneca (Vaxzevria), seguidas da declaração pela EMA, a 18 de março, garantindo que o número de eventos observados não seria superior ao esperado e aconselhando a continuação da vacinação com a Vaxzevria, uma vez que os benefícios superariam os riscos. ${ }^{4}$ Pouco tempo depois, três grupos independentes, da Noruega, ${ }^{5}$ Alemanha/Áustria, ${ }^{6}$ e Reino Unido, ${ }^{7}$ descreveram um total de 39 pessoas com um novo síndrome caracterizado por trombose, trombocitopenia e presença de anticorpos circulantes contra o fator plaquetário 4 (FP4). ${ }^{8}$ Estes doentes, $66,7 \%$ mulheres e média etária de 42,5 anos (a variar entre 21 e 77 anos), foram admitidos no hospital cinco a 24 dias após vacinação, com tromboses atípicas em localização não usual, com predomínio das tromboses dos seios venosos cerebrais (TSVC) em dois terços dos casos e tromboses esplâncnicas. Tromboses típicas como trombose venosa profunda (TVP) ou embolia pulmonar (EP) assim como eventos arteriais, também foram descritos..$^{5-8}$ Dois destes doentes apresentaram trombocitopenia sem trombose (um deles faleceu com uma hemorragia cerebral sem que uma TSVC pudesse ter sido excluída, ${ }^{6}$

1. Unidade de Trombose e Hemostase. Serviço de Hematologia Clínica. Hospital de Santo António. Centro Hospitalar Universitário do Porto. Porto. Portugal.

2. Unidade Multidisciplinar de Investigação Biomédica. Instituto de Ciências Biomédicas. Universidade do Porto. Porto. Portugal.

3. Basic and Clinical Research on Iron Biology Group. Instituto de Investigação e Inovação em Saúde - i3S. Universidade do Porto. Porto. Portugal.

$\triangle$ Autor correspondente: Sara Morais. saratsmorais@hotmail.com

Recebido: 24 de maio de 2021 - Aceite: 16 de junho de 2021 - First published: 18 de junho de 2021 - Online issue published: 01 de setembro de 2021 Copyright $\odot$ Ordem dos Médicos 2021 
e um outro apresentou apenas sintomas hemorrágicos ${ }^{7}$ ). A mortalidade foi superior à esperada (aproximadamente $40 \%$ ) como consequência da lesão isquémica cerebral e/ ou hemorragia cerebral. ${ }^{9} \mathrm{O}$ número de plaquetas ao diagnóstico variou entre cerca de 10000 e 110 000/. $\mathrm{mm}^{3}$, e os níveis de D-dímeros apresentavam-se acentuadamente aumentados acompanhados por níveis baixos a normais de fibrinogénio. ${ }^{8}$ Em quase todos os doentes foram identificados níveis elevados de anticorpos anti-FP4, detetados por testes de ELISA usados na suspeita de trombocitopenia induzida pela heparina ( $\mathrm{TIH})$, mas outros testes usados por rotina (quimioluminiscência ou imunoensaios por látex) são frequentemente negativos. ${ }^{8}$

Esta combinação não usual de trombocitopenia e trombose, em particular TSVC, que partilha muitas semelhanças com a TIH, é uma condição altamente pró-trombótica e com mortalidade elevada, tendo sido proposta a designação de Vaccine-induced Immune Thrombocytopenia and Thrombosis ou Vaccine-induced Immune Thrombotic Thrombocytopenia (VITT). ${ }^{8}$ Ao contrário da TIH, na VITT a formação dos anticorpos anti-FP4 ocorre na ausência de exposição à heparina, mimetizando uma entidade previamente descrita ${ }^{10}$ denominada TIH autoimune ou atípica, na qual a trombose surge na ausência de exposição prévia conhecida à heparina.
Mais recentemente, surgiu a publicação de um caso de VITT 14 dias após vacinação com a vacina Ad26.COV2.S da Johnson \& Johnson/Janssen ( $\mathrm{VJ} \& \mathrm{~J}),{ }^{11}$ seguido pelo relato de 12 outros casos com o mesmo fenótipo clínico e laboratorial dos casos descritos após vacinação com a Vaxzevria. ${ }^{12}$

São vários os casos revistos pelo Comité de Segurança da EMA associados à Vaxzevria (em 4 de abril, um total de 169 casos de TSVC e 53 casos de tromboses venosas esplâncnicas). ${ }^{13}$ Muitos outros têm sido reportados associados à vacinação com as vacinas RNAm (Pfizer- BioNTech e Moderna), embora nem todos tenham sido revistos centralmente ou haja informação sobre a avaliação dos anticorpos anti-FP4. ${ }^{9} \mathrm{~A}$ taxa de incidência estimada de VITT é de cerca de 1 caso por 100000 vacinações, ${ }^{9}$ a variar entre 1 caso por 26500 e 1 caso por 127300 vacinações. ${ }^{14}$ Pensa-se, no entanto, que a taxa de incidência esteja subestimada, devido à não obrigatoriedade da notificação.

\section{RECOMENDAÇÕES}

O reconhecimento desta nova entidade, levou ao rápido aparecimento de protocolos de diagnóstico e orientação clínica de diferentes sociedades científicas nacionais e internacionais (Fig. 1). ${ }^{15-21}$ Da síntese dessas orientações, sugerimos as seguintes recomendações:

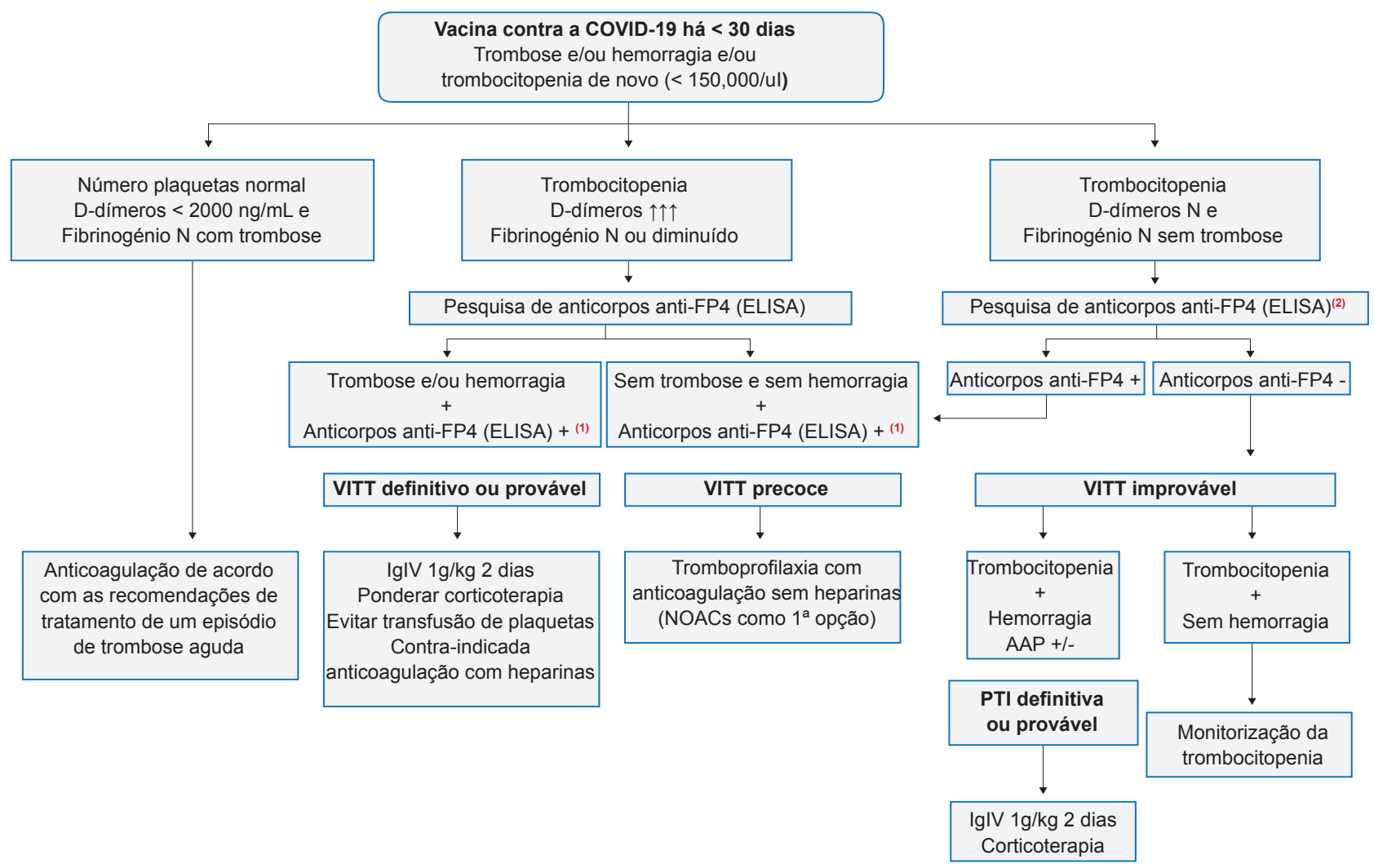

Figura 1 - Estratégias de diagnóstico e tratamento da trombocitopenia, trombose e/ou hemorragia, associada à vacinação contra a COVID-19

VITT: vaccine-induced Immune Thrombocytopenia and Thrombosis; PTI: púrpura trombocitopénica imune; FP4: fator plaquetário 4; IgIV: imunoglobinas intra-venosas; AAP: anticorpos antiplaquetários.

(1): na ausência de resultado de anticorpos anti-FP4, assumir VITT provável.

(2): não obrigatória pesquisa de anti-corpos anti-FP4, nesta situação. 
1. Até 30 dias após vacinação, em particular com as vacinas Vaxzevria e $v J \& J$, deve ser procurada assistência médica imediata na presença de ${ }^{13,15,8,20}$ :

- sintomas neurológicos, incluindo tonturas, dores de cabeça graves e persistentes ou visão turva;

- dor abdominal persistente;

- falta de ar ou dor no peito;

- edema ou dor persistente nos membros;

- presença de petéquias ou equimoses para além do local da injeção.

Sintomas semelhantes aos da gripe, como artralgias, mialgias ou cefaleias, que persistem por um a dois dias após a vacinação, são efeitos comuns e não são motivo de preocupação. ${ }^{13,15}$

2. Em presença dos sintomas referidos, devem ser realizados estudos de imagem apropriados para diagnóstico de trombose aguda e avaliação analítica, que inclui hemograma completo com contagem de plaquetas e esfregaço de sangue periférico (para confirmação de trombocitopenia), e estudo de coagulação com D-dímeros e fibrinogénio. 15, 17-19 3. Perante episódio de trombose aguda confirmado, com contagens de plaquetas normais, D-dímeros $<2000 \mathrm{ng} /$ $\mathrm{mL}$ e fibrinogénio normal, é possível excluir a VITT. O tratamento é o tratamento anticoagulante de qualquer outro episódio agudo de trombose.

4. Em presença de trombocitopenia e evidência de trombose aguda e/ou hemorragia deve ser efetuada pesquisa de anticorpos anti-FP4 ${ }^{15-21}$ :

- Indicados testes por ELISA para deteção de TIH (baseados na deteção imunológica de anticorpos contra o complexo FP4/heparina). ${ }^{15-19}$

- Nem todos os testes comerciais para diagnóstico de TIH podem ser usados (não estão indicados testes por outros métodos, como Acustar ou o método latex). ${ }^{15,16,19,21}$

- Testes funcionais anti-FP4, se disponíveis, ajudam a diferenciar uma TIH autoimune de VITT, ${ }^{15,19}$ embora a sua utilidade não seja muito clara. ${ }^{12}$

- Na ausência de disponibilidade imediata de um teste anti-FP4, a amostra de sangue periférico colhida antes do tratamento, deve ser congelada, para posterior deteção de anticorpos. ${ }^{16}$

5. Diagnóstico definitivo de VITT, se trombocitopenia, D-dímeros muito aumentados e trombose progressiva, com uma grande preponderância de TSVC; a hemorragia também pode ser significativa e inesperada. ${ }^{16}$

- Tipicamente contagem de plaquetas inferior a 150 x 10\%, níveis muito aumentados de D-dímeros (> $4000 \mathrm{ng} / \mathrm{mL}$ ) e em alguns casos níveis baixos de fibrinogénio; foi reconhecido recentemente que até $5 \%$ dos doentes podem ter contagens de plaquetas normais na apresentação, podendo desenvolver trombocitopenia nos dias subsequentes. ${ }^{16}$

- Presença de anticorpos anti-FP4 (por testes ELISA) na ausência de exposição à heparina.

6. Diagnóstico provável de VITT, se trombose e/ou hemorragia, trombocitopenia e D-dímeros muito aumentados e níveis de fibrinogénio normais ou baixos. $\mathrm{Na}$ ausência de disponibilidade imediata para teste ELISA anti-FP4 tratar como VITT, enquanto se aguarda confirmação de diagnóstico. ${ }^{16}$

7. O tratamento do VITT definitivo ou provável passa por $^{15,16,19,21}$ :

- Tratamento imediato com IgIV, mesmo na ausência de confirmação do diagnóstico $(1 \mathrm{~g} / \mathrm{kg} /$ dia durante dois dias). Podem ser necessárias doses adicionais.

- Evitar transfusão de plaquetas. Se necessário procedimento neurocirúrgico, as plaquetas devem ser efetuadas durante ou após administrar IgIV. ${ }^{16}$

- Evitar qualquer administração de heparina (incluindo nos cateteres) pela possibilidade teórica de exacerbar a VITT.

- Anticoagulação com anticoagulantes sem heparina [fondaparinux, argatrobano - utilizado no tratamento da trombocitopenia induzida pela heparina em hospitais portugueses, por importação direta, e noutros hospitais europeus apesar de não estar aprovado pela EMA - danaparóide, anticoagulantes orais diretos (DOAC)] com doses ajustadas de acordo com a trombocitopenia ${ }^{19}$

\section{Se Plaquetas $>100 \times 10^{\circ} / \mathrm{L}$}

Fondaparinux: 5/7,5/10 mg para peso corporal < $50 / 50-100 />100 \mathrm{~kg}$, respetivamente.

Argatrobano: rácio de TTPA 1,5-2,5

\section{Se Plaquetas 50 - 100x 10\%/L}

Fondaparinux: 5/7,5 mg para peso corporal $<50$ ou $>50 \mathrm{~kg}$

Argatrobano: rácio de TTPA 1,5-2,5

Se Plaquetas 20 - 50x 10\%

Fondaparinux: $2,5 / 5 \mathrm{mg}$ para peso corporal $<50$ ou $>50 \mathrm{~kg}$

Argatrobano: rácio de TTPA 1,5

\section{Se Plaquetas < 20 - 50x 10\%}

Evitar anticoagulação.

- Podem estar indicados corticoides, sobretudo se houver atraso na administração de IgIV. ${ }^{16,19,20} \mathrm{Se} 0$ valor de plaquetas $<20 \times 10^{\circ} / \mathrm{L}$, está indicado associar dexametasona $40 \mathrm{mg} /$ dia durante quatro dias ${ }^{19}$ ou prednisolona $1 \mathrm{mg} / \mathrm{kg} / \mathrm{dia}$, se plaquetas $<50 \mathrm{x}$ $10^{9} /$ L. $^{20}$

- Considerar suplementação com concentrados de fibrinogénio, se níveis plasmáticos de fibrinogénio < $1,5 \mathrm{~g} / \mathrm{L}$.

- Considerar plasmaférese se se verificar deterioração clínica apesar do tratamento. No caso de trombose extensa, trombocitopenia $<30 \times 10^{9} / \mathrm{L}$ ou níveis muito elevados de anticorpos anti-FP4, a plasmaférese deve ser considerada precocemente. ${ }^{16}$

- Não está recomendada antiagregação plaquetária.

- A presença de trombose complicada por hemorragia, em particular em doentes com TSVC, torna o tratamento um desafio. Não se deve introduzir anticoagulação enquanto houver hemorragia ativa. 
- Em caso de trombose arterial, é preferida a anticoagulação sobre a antiagregação até à normalização dos valores de plaquetas, D-dímeros e fibrinogénio; só então é que se deve mudar para terapêuticas antiplaquetárias. ${ }^{16}$

8. Trombocitopenia sem trombose, com D-dímeros normais ou ligeiramente aumentados e fibrinogénio normal, torna o diagnóstico de VITT improvável. ${ }^{16,21}$

- Se trombocitopenia sem hemorragia, devem-se monitorizar continuamente os parâmetros clínicos e laboratoriais. ${ }^{21}$

- Trombocitopenia e hemorragia aponta para trombocitopenia imune (PTI), a confirmar pela presença de anticorpos antiplaquetários (AAP). Está indicado IgIV e/ou corticoides mas não anticoagulação. ${ }^{21}$

9. Trombocitopenia sem hemorragia ou trombose, mas com alterações nos parâmetros da coagulação (D-dímeros aumentados) pode indiciar uma VITT precoce, estando indicado iniciar tromboprofilaxia com anticoagulantes sem heparinas. ${ }^{21}$

10. História prévia de trombose ou trombofilia conhecida não são considerados fatores de risco para o desenvolvimento de VITT. Não há evidência de que tromboses em localizações típicas (TVP, EP) sejam mais comuns após vacinação do que na população em geral do mesmo grupo etário. ${ }^{15}$

11. Não está indicada profilaxia de rotina com anticoagulantes ou antiplaquetários na prevenção das tromboses atípicas associadas à vacinação. ${ }^{15}$

- Doentes a fazer anticoagulação oral por outras indicações [ex: fibrilação auricular ou tromboembolismo venoso (TEV)] devem continuar a anticoagulação durante e após vacinação. ${ }^{15}$

- Em doentes sem indicação para anticoagulação oral, mas em risco significativo de TEV (ex: sintomas gripais com febre e imobilização), pode estar indicada tromboprofilaxia farmacológica, avaliada numa base individual. ${ }^{15}$

- Se a tromboprofilaxia estiver indicada, considerar doses profiláticas de DOAC (rivaroxabano $10 \mathrm{mg} /$ dia ou apixabano 2,5 mg 12/12 horas). Não está recomendada profilaxia com heparinas de baixo peso molecular (HBPM). ${ }^{15}$

12. Todos os casos de VITT, trombose, hemorragia ou trombocitopenia, 30 dias após administração de vacinas anti COVID-19, devem ser reportados, independentemente da probabilidade de associação com a vacinação. ${ }^{16}$

\section{CONTRIBUTO DOS AUTORES}

Ambos os autores contribuíram de igual forma para a concepção do artigo, revisão da literatura e redação do manuscrito.

\section{CONFLITOS DE INTERESSE}

Os autores declaram não ter conflitos de interesses relacionados com o presente trabalho.

\section{FONTES DE FINANCIAMENTO}

Este trabalho não recebeu qualquer tipo de suporte financeiro de nenhuma entidade no domínio público ou privado.

\section{REFERÊNCIAS}

1. Lee EJ, Cines DB, Gernsheimer T, Kessler C, Michel M, Tarantino MD, et al. Thrombocytopenia following Pfizer and Moderna SARS-CoV-2 vaccination. Am J Hematol. 2021;96:534-7.

2. Merchant HA. CoViD vaccines and thrombotic events: EMA issued warning to patients and healthcare professionals. J Pharm Policy Pract. 2021;14:32

3. Perricone C, Ceccarelli F, Nesher G, Borella E, Odeh Q, Conti F, et al. Immune thrombocytopenic purpura (ITP) associated with vaccinations: a review of reported cases. Immunol Res. 2014;60:226-35.

4. European Medicines Agency. COVID-19 vaccine AstraZeneca: benefits still outweigh the risks despite possible link to rare blood clots with low blood platelets. March 18, 2021 [consultado 2021 mar 21] Disponível em: https://www.ema.europa.eu/en/news/ covid-19-vaccineastrazeneca-benefits-still-outweigh-risks-despite-possible-link-rareblood-clots.

5. Schultz NH, Sørvoll IH, Michelsen AE, Munthe LA, Lund-Johansen F, Ahlen MT, et al. Thrombosis and thrombocytopenia after ChAdOx1 nCoV-19 vaccination. N Engl J Med. 2021;384:2124-30.

6. Greinacher A, Thiele T, Warkentin TE, Weisser K, Kyrle PA, Eichinger S. Thrombotic thrombocytopenia after ChAdOx1 nCov-19 vaccination. N Engl J Med. 2021;384:2092-101.

7. Scully M, Singh D, Lown R, Poles A, Solomon T, Levi M, et al. Pathologic antibodies to platelet factor 4 after ChAdOx1 $\mathrm{nCoV}-19$ vaccination. $\mathrm{N}$ Engl J Med. 2021;384:2202-11.

8. Makris M, Pavord S, Lester W, Scully M, Hunt BJ. Vaccine-induced Immune Thrombocytopenia and Thrombosis (VITT). Res Pract Thromb Haemost. 2021:5:e12529.

9. Cines DB, Bussel JB. SARS-CoV-2 Vaccine-induced immune thrombotic thrombocytopenia. N Eng J Med. 2021; 384:2254-6.

10. Greinacher A, Selleng K, Warkentin TE. Autoimmune heparin-induced

thrombocytopenia. J Thromb Haemost. 2017;15:2099-114.

11. Muir KL, Kallam A, Koepsell SA, Gundabolu K. Thrombotic thrombocytopenia after AD26.COV2.S vaccination. N Engl J Med 2021. 384:1964-5.

12. See I, Su JR, Lale A, Woo EJ, Guh AY, Shimabukuro TT, et al. US case reports of cerebral venous sinus thrombosis with thrombocytopenia after Ad26.COV2.S vaccination, March 2 to April 21, 2021. JAMA. 2021;e217517.

13. European Medicines Agency. AstraZeneca's COVID-19 vaccine: EMA finds possible link to very rare cases of unusual blood clots with low blood platelets. April 7, 2021. [consultado 2021 abr 10]. Disponível em: https://www.ema.europa.eu/en/news/astrazenecas -covid-19-vaccine -ema-finds-possible-link-very-rare-cases-unusual-blood-clots-lowblood

14. Chan B, Odutayo A, Jüni $P$, Stall NM, Bobos $P$, Brown AD, et al. Risk of vaccine-induced thrombotic thrombocytopenia (VITT) following the AstraZeneca/COVISHIELD adenovirus vector COVID-19 vaccines. Science Briefs of the Ontario COVID-19 Science Advisory Table. Ontario: Ontario COVID-19 Science Advisory Table; 2021.

15. Oldenburg J, Klamroth R, Langer F, Albisetti M, von Auer C, Ay C, et al. Diagnosis and management of vaccine-related thrombosis following AstraZeneca COVID-19 vaccination: Guidance Statement from the GTH. Hamostaseologie. 2021 (in press). doi: 10.1055/a-1469-7481

16. British Society for Haematology. Guidance produced from the Expert Haematology Panel (EHP) focussed on Covid-19 Vaccine induced Thrombosis and Thrombocytopenia (VITT). Updated Guidance on Management. Version 2.0 28 May 2021 [consultado 2021 junho 16]. Disponível em: https://b-s-h.org.uk/media/19718/ guidance-v20-20210528-002.pdf

17. Australian Government Department of Health. ATAGI statement on 
AstraZeneca vaccine in response to new vaccine safety concerns. A statement from the Australian Technical Advisory Group on Immunisation (ATAGI) on the AstraZeneca COVID-19 vaccine in response to new vaccine safety concerns. April 8, 2021. [consultado 2021 abr 9]. Disponivel em: https://www.health.gov.au/news/atagi-statement-onastrazeneca-vaccine-in-response-to-new-vaccine-safety-concerns.

18. Thrombosis Canada. Vaccine-Induced Prothrombotic Immune Thrombocytopenia (VIPIT). 26 April 2021. [consultado $2021 \mathrm{abr}$ 28]. Disponível em: https://thrombosiscanada.ca/wp-uploads/ uploads/2021/04/51.-Vaccine-induced-prothrobotic-immunethrombcytopenia_26Apr21-Final.pdf.

19. Gresele P, Marietta M, Ageno W, Marcucci R, Contino L, Donadini MP, et al. Management of cerebral and splanchnic vein thrombosis associated with thrombocytopenia in subjects previously vaccinated with Vaxzevria
(AstraZeneca): a position statement from the Italian Society for the Study of Haemostasis and Thrombosis (SISET). Blood Transfus. 2021 (in press). doi: 10.2450/2021.0117-21.

20. International Society on Thrombosis and Haemostasis. ISTH Interim Guidance for the Diagnosis and Treatment on Vaccine Induced Immune Thrombotic Thrombocytopenia (Updated 20 April, 2021). [consultado 2021 abr 21]. Disponível em: https://cdn.ymaws.com/www.isth.org/ resource/resmgr/ISTH_VITT_Guidance_2.pdf

21. Nazy I, Sachs UJ, Arnold DM, McKenzie SE, Choi P, Althaus K, et al. Recommendations for the clinical and laboratory diagnosis of vaccineinduced immune thrombotic thrombocytopenia (VITT) for SARS-CoV-2 infections: Communication from the ISTH SSC Subcommittee on Platelet Immunology. J Thromb Haemost. 2021;19:1585-8. 